\title{
Tetraspanin Protein CD9 Is a Novel Paranodal Component Regulating Paranodal Junctional Formation
}

\author{
Tomoko Ishibashi, ${ }^{1}$ Lei Ding, ${ }^{2}$ Kazuhiro Ikenaka, ${ }^{3}$ Yoshiro Inoue, ${ }^{2}$ Kenji Miyado, ${ }^{4}$ Eisuke Mekada, ${ }^{4}$ and Hiroko Baba ${ }^{1}$ \\ ${ }^{1}$ Department of Molecular Neurobiology, School of Pharmacy, Tokyo University of Pharmacy and Life Science, Hachioji 192-0392, Japan, ${ }^{2}$ Department of \\ Molecular Neuroanatomy, Hokkaido University School of Medicine, Sapporo 060-8368, Japan, ${ }^{3}$ Laboratory of Neural Information, National Institute for \\ Physiological Sciences, Okazaki National Research Institutes, Okazaki 444-8585, Japan, ${ }^{4}$ and Department of Cell Biology, Research Institute for Microbial \\ Disease, Osaka University, Suita 565-0871, Japan
}

The axoglial paranodal junction is essential for the proper localization of ion channels around the node of Ranvier. The integrity of this junction is important for nerve conduction. Although recent studies have made significant progress in understanding the molecular composition of the paranodal junction, it is not known how these membrane components are distributed to the appropriate sites and interact with each other. Here we show that CD9, a member of the tetraspanin family, is present at the paranode. CD9 is concentrated in the paranode as myelination proceeds, but CD9 clusters become diffuse, associated with disruption of the paranode, in cerebroside sulfotransferase-deficient mice. Immunohistochemical and Western blot analysis showed that CD9 is distributed predominantly in the PNS. Ablation of CD9 in mutant mice disrupts junctional attachment at the paranode and alters the paranodal components contactinassociated protein (also known as Paranodin) and neurofascin 155, although the frequency of such abnormalities varies among individuals and individual axons even in the same mouse. Electron micrographs demonstrated that compact myelin sheaths were also affected in the PNS. Therefore, CD9 is a myelin protein important for the formation of paranodal junctions. CD9 also plays a role in the formation of compact myelin in the PNS.

Key words: CD9; tetraspanin-TM4SF; myelin; paranode; axoglial interaction; node of Ranvier

\section{Introduction}

In myelinated axons, the lateral edge of myelin sheath spirals around the axon at either end of each node of Ranvier, forming the axoglial junctions at the paranodes. Morphologically, paranodal junctions are similar to septate junctions, which display a characteristic array of ladder-like cross-bridges found in invertebrate epithelial cells and glia (Rosenbluth, 1976; Pedraza et al., 2001). These junctions are thought to have three functional roles: electrical insulation, acting as a barrier to surface proteins on the axons, and mediating signals between axon and glia. Recent studies have made significant progress in understanding the molecular composition of the paranodal junction. Contactin-associated protein (Caspr) (also known as Paranodin), the mammalian homolog of Drosophila neurexin IV (Baumagartner et al., 1996), is

Received May 16, 2003; revised 0ct. 29, 2003; accepted 0ct. 31, 2003.

This work was supported by a grant for private universities provided by the Ministry of Education, Culture, Sports, Science, and Technology and the Promotion and Mutual Aid Corporation for Private Schools of Japan (H.B.) and by Grants-in-Aid 14580749 (K.I., H.B.), 10214204, and 13480270 (H.B., T.I., K.I.) from the Ministry of Education, Culture, Sports, Science, and Technology of Japan. The monoclonal and polyclonal Caspr antibodies were kind gifts from Dr. Elior Peles (The Weizmann Institute of Science, Rehovot, Israel). CST-deficient mice were kindly provided by Dr. Kouichi Honke (Osaka University, Osaka, Japan). We thank Drs. Steve E. Pfeiffer (University of Connecticut Medical School, Farmington, CT) and Hitoo Nishino (Nagoya City University Medical School, Nagoya, Japan) for helpfu discussion. The microscopic observations reported in this paper were obtained using microscopes in the electron microscopy room at the National Institute for Physiological Sciences.

Correspondence should be addressed to Dr. Hiroko Baba, Department of Molecular Neurobiology, School of Pharmacy, Tokyo University of Pharmacy and Life Science, 1432-1 Horinouchi, Hachioji, Tokyo 192-0392, Japan. E-mail: hirobaba@ps.toyaku.ac.jp.

DOI:10.1523/JNEUROSCI.1484-03.2004

Copyright $\odot 2004$ Society for Neuroscience $\quad$ 0270-6474/04/240096-07\$15.00/0 expressed only in neurons and is found exclusively in the paranodal junctions in mature myelinated axons (Einheber et al., 1997; Menegoz et al., 1997; Peles et al., 1997). Its binding partner contactin, a glycosylphosphatidylinositol-anchored protein, is also expressed in neurons and localized in the paranodal junction (Rios et al., 2000). Direct interaction between Caspr and contactin is required for the cell-surface expression of Caspr (FaivreSarrailh et al., 2000). Neurofascin 155 (NF155), an ankyrinbinding member of the L1 family, was identified as the first glial paranodal molecule (Tait et al., 2000). In addition, one of the myelin glycolipids, sulfatide, is also considered to be essential for paranodal junction formation (Honke et al., 2002; Ishibashi et al., 2002). Although NF155 may be a counterpart of the Caspr-contactin complex (Charles et al., 2002), the possibility of the presence of another glial component that interacts with the Casprcontactin complex cannot be excluded. The mechanisms underlying proper localization of these membrane components and their interaction are not known.

The tetraspanins [also referred to as transmembrane- 4 super family (TM4SF)] are characterized as having four transmembrane domains with cytoplasmic $\mathrm{N}$ and $\mathrm{C}$ termini and the conserved cysteine motif (Wright and Tomlinson, 1994; Maecker et al., 1997; Hemler, 2001), which act as "molecular facilitators" and linkers for transmembrane proteins forming a tetraspanin web (Hemler, 2001). One of the tetraspanins, CD9, is a $24-27 \mathrm{kDa}$ cell-surface glycoprotein (Boucheix and Benoit, 1988) expressed in a wide variety of cells, which is also known as diphtheria toxin receptor-associated protein (Iwamoto et al., 1991). A number of 


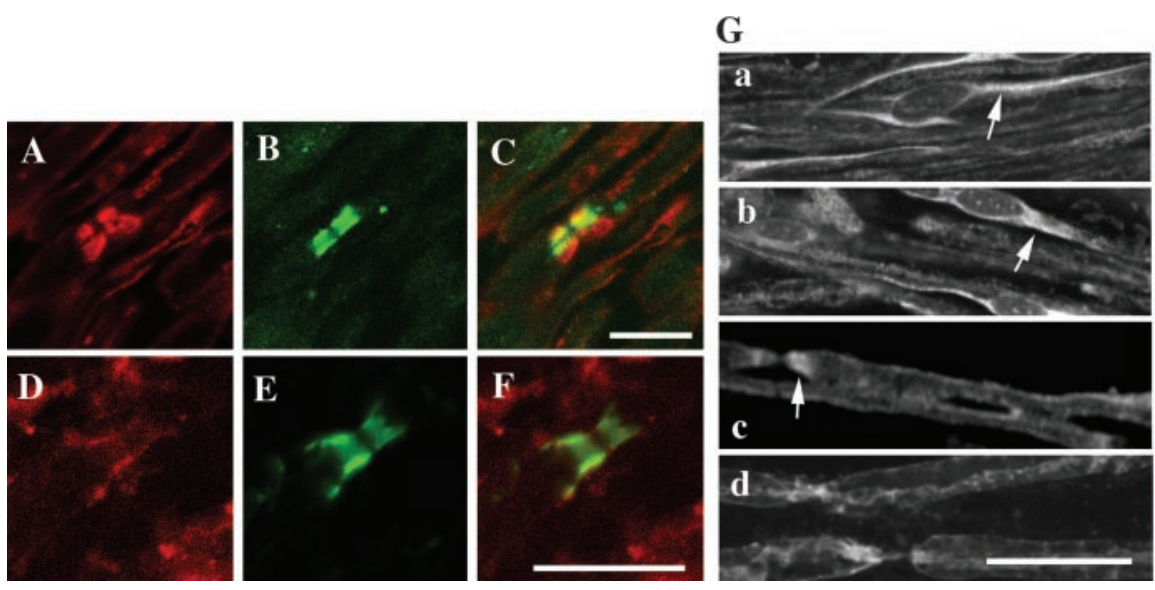

Figure 1. Localization of $C D 9$ in longitudinal sections of the nervous system. $A-F$, The localization of $C D 9$ in longitudinal sections of 8-week-old mouse sciatic nerve $(A-C)$ and cervical ventral spinal cord $(D-F)$. Immunoreactivity of $C D 9$ shows a pair of clusters $(A)$. From double immunostaining, $C D 9$ (red) is colocalized with Caspr (green) at the paranodes ( $)$. CD9 (D) and Caspr $(E)$ are also colocalized at the paranodes $(F)$ in the spinal cord. $G$, Developmental profile of $C D 9$ expression in sciatic nerve. At PO (Ga) and P1 (Gb), CD9 is diffusely localized in Schwann cell bodies (arrows in Ga and Gb). At P2 (Gc), CD9 starts to redistribute not only to the outer surface of myelin but also to the paranodal region as myelination proceeds (indicated by the arrow). At P7 (Gd), CD9 is concentrated at the paranode. Scale bars, $10 \mu \mathrm{m}$.

studies have suggested that CD9 is involved in a variety of cell processes, including cell signaling, adhesion, motility, and tumor cell metastasis (for review, see Maecker et al., 1997). Because CD9 appears at the final stage of myelination (Kagawa et al., 1997), it is a good candidate for involvement in communication between axons and glia.

In this paper, we report the role of CD9 in formation of axoglial paranodal junctions. We observed that CD9 and Caspr are colocalized at the paranode both in the CNS and in the PNS. On the basis of analysis of mice lacking CD9, we provide evidence that CD9 has a significant role to play at the paranode in the CNS and PNS and has additional influence on the formation of compact myelin in the PNS.

\section{Materials and Methods}

Antibodies. The polyclonal antibody against $\mathrm{Na}^{+}$channel has been described previously (Ishibashi et al., 2002). The polyclonal antibody against NF155 was generated against the synthetic peptides of the second to the third FNIII domain specific to NF155 (CLWVSQKRQQASFPGDRPR) (Tait et al., 2000) and used at a dilution of 1:200. A mouse monoclonal antibody, $\mathrm{K} 58 / 35$, against $\mathrm{Na}^{+}$channel (anti-pan $\mathrm{Na}^{+}$channel antibody) was purchased from Sigma (St. Louis, MO) and used at a dilution of 1:1000. The polyclonal and monoclonal antibodies against $\mathrm{K}^{+}$channel $\alpha$-subunits (Trimmer, 1991) were kindly provided by Dr. J. S. Trimmer (State University of New York, Stony Brook, NY) and used at a dilution of 1:100. The polyclonal and monoclonal anti-Caspr antibodies were a gift from Dr. Elior Peles and used at a dilution of 1:2500 and of 1:250, respectively (Peles et al., 1997). Anti-mouse CD9 hybridoma (KMC8.8) was a kind gift from Dr. Y. Nagai (Tokyo University, Tokyo, Japan) and was used at a dilution of 1:50. Antibody to Kv1.1 $\mathrm{K}^{+}$channel (monoclonal; Upstate Biotechnology, Lake Placid, NY) was used at a dilution of 1:200.

Animals. CD9-deficient mice (Miyado et al., 2000) and cerebroside sulfotransferase (CST)-deficient mice (Honke et al., 2002) were produced by homologous recombination as described previously. CD9-deficient mice were originally generated on the $129 / \mathrm{Sv}$ genetic background, and chimeric offspring were mated with C57BL/6J females. The heterozygous mice were mated with $\mathrm{C} 57 \mathrm{BL} / 6 \mathrm{~J}$ mice to produce homozygous mutants. $\mathrm{F}_{2}-\mathrm{F}_{4}$ generations of C57BL/6J homozygous and littermate control mice were used for the present study. Genotypes of pups from mating heterozygous mice were determined by PCR using specific primer sets. These mice were maintained in the animal facility at the Tokyo University of Pharmacy and Life Science. Animals were maintained according to the National Institutes of Health Guide for the Care and Use of Laboratory Animals, and experiments were performed to the animal use committee protocol approval of the university.

Immunohistochemistry. Homozygotic CSTdeficient and CD9-deficient mice as well as their wild-type controls were fixed by transcardial perfusion with $4 \%$ paraformaldehyde in 0.1 M phosphate buffer ( $\mathrm{PB}), \mathrm{pH}$ 7.4. The optic nerves, cervical (C3-C5) spinal cords, and sciatic nerves were cryoprotected with $30 \%$ sucrose in PBS, pH 7.4, for $24 \mathrm{hr}$ at $4^{\circ} \mathrm{C}$. Teased nerve fibers were prepared from unfixed sciatic nerves as described previously (Rasband et al., 1998). After embedding in optimal cutting temperature mounting medium (Miles, Elkhart, IN), tissues were cut into $7-\mu \mathrm{m}$-thick sections. The sections were collected on 3-aminopropyltriethoxysilane (Sigma) rubbed glass slides and allowed to air dry. Immunofluorescence staining was performed as described previously (Ishibashi et al., 2002). Secondary antibodies were Alexa 488 or Alexa 594 conjugated to goat anti-rabbit, goat anti-rat, or goat anti-mouse antibodies (Molecular Probes, Eugene, OR). Images were captured with either laser scanning microscopes LSM510 or Pascal (Zeiss, Oberkochen, Germany). Digitized images were transferred to a laboratory computer for analysis using Adobe Photoshop 6.0 (Adobe Systems, San Jose, CA). For quantification of Caspr clusters, we used criteria described previously (Ishibashi et al., 2002).

Electron microscopic analysis. Mice were deeply anesthetized with ether and transcardially perfused with a mixture of $4 \%$ paraformaldehyde and $0.5 \%$ glutaraldehyde in $0.1 \mathrm{M} \mathrm{PB}$ at $\mathrm{pH}$ 7.4. Dissected tissues were immersed in the same fixative overnight, washed with PBS, and postfixed with $1 \%$ osmium tetroxide. After fixation, the tissues were washed again with PBS, dehydrated, and embedded in Epon. Ultrathin sections were cut and stained with $2 \%$ uranyl acetate aqueous solution and Sato's mixed lead solution (Sato, 1968).

Western blot analysis. The 8-week-old control mouse brain myelin fraction was purified and extracted as described previously (Huber et al., 1994; Kim et al., 1995) on the basis of the procedures of Norton and Poduslo (1973). The 8-week-old mouse sciatic nerves were harvested immediately, placed in ice-cold PBS, and ground into pieces with a mortar in liquid nitrogen. The sciatic nerves were then placed into $0.32 \mathrm{M}$ sucrose in $5 \mathrm{~mm}$ Tris$\mathrm{HCl}, \mathrm{pH}$ 7.4, and homogenized by Polytron (Heidolph, Schwabach, Germany). The protein concentration of the lysates was determined by protein assay (Bio-Rad, Hercules, CA). The samples were analyzed by SDS-PAGE and electroblotted to Hybond-P transfer membrane (Amersham Biosciences, Buckinghamshire, UK). The blots were blocked for $1 \mathrm{hr}$ in $5 \%$ nonfat milk containing $10 \mathrm{~mm}$ Tris- $\mathrm{HCl}, 100 \mathrm{~mm} \mathrm{NaCl}$, and $0.1 \%$ Tween 20 $\mathrm{pH} 7.5$ (blocking buffer) at room temperature (RT). Then they were probed with antibody specific for CD9 (1:5000) with blocking buffer for $1 \mathrm{hr}$ at RT. After incubation with an HRP-conjugated second antibody diluted in blocking buffer for $30 \mathrm{~min}$ at RT, the blots were detected using the ECL system (Amersham Biosciences).

\section{Results}

\section{CD9 is concentrated in the paranodal region}

CD9 was characterized previously as a myelin protein located in the outermost membrane of compact myelin (Nakamura et al., 1996). In this study, the localization of CD9 in central and peripheral myelin was examined in detail by double immunostaining using specific antibodies to CD9 and Caspr (Fig. 1). The immunoreactivity of CD9 showed a pair of clusters in the adult sciatic nerve (Fig. 1A,C). These clusters were colocalized with Caspr (Fig. $1 B, C$ ), an axonal paranodal protein, indicating that 

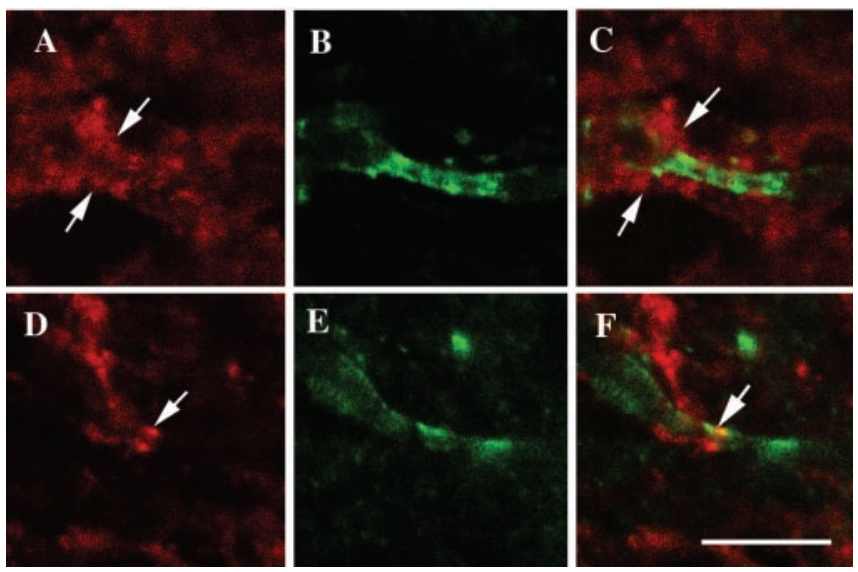

Figure 2. Altered localization of CD9 in CST-deficientmice. Shown is double immunostaining with CD9 (red) and Caspr (green) of the cervical ventral spinal cord from 22-week-old CST-deficient mice. Caspr clusters lose their tight association in the paranodal regions $(B, E)$, and characteristic clusters of CD9 disappear ( $A$, C, arrows) in CST-deficient mice. Occasionally, the colocalization of these proteins at the presumptive paranode is observed in the mutants $(D, F$, arrow). Scale bar, $10 \mu \mathrm{m}$.

CD9 was concentrated at the paranode in addition to the outermost myelin membrane. In contrast, in the CNS, such characteristic staining of CD9 was not as prominent as it was in the PNS. However, as shown in Figure 1D-F, CD9 (red) was also colocalized with Caspr (green) at the paranodal region in the mouse cervical ventral spinal cord. Thus, these results identify CD9 as a newly recognized paranodal myelin protein both in the CNS and in the PNS.

To understand when CD9 is accumulated in the paranodal regions, we examined CD9 expression during development using teased fibers from a mouse sciatic nerve. At postnatal day 0 (P0) and P1, before myelin ensheathment, CD9 first appeared in the cell body of the Schwann cells (Fig. $1 G a, b$, arrows). At P2, when some axons began to myelinate, CD9 began to accumulate in the bilateral ends of myelin (Fig. 1Gc, arrow) as well as at the myelin surface. By P7, clustering at the paranode became more pronounced as the myelin matured (Fig. $1 G d$ ). These results clearly indicated that CD9 was progressively concentrated into the paranodal region correlated with formation of the paranodal junction during myelination.

\section{CD9 is mislocalized in the paranodal mutant}

To determine whether CD9 is localized in the junctional surface of the paranode, we investigated how the disruption of the paranodal junction affects the localization of CD9. CST-deficient mice lack sulfatide and display mislocalization of paranodal proteins (Ishibashi et al., 2002) with disruption of the paranodal junction (Honke et al., 2002). The localization of CD9 was examined in the spinal cords from the mutants and wild-type controls. To define the normal paranodal junction, double immunostaining for Caspr and CD9 was performed. As described in Figure 1, in wild-type mice, CD9 was concentrated at the paranodal region where Caspr was clustered (Fig. 1D-F). In contrast, in CSTdeficient mice, wherever the specific localization of Caspr disappeared in the disrupted paranodal junction (Fig. $2 B, C$ ), CD9 became disordered (Fig. $2 A, C$ ). The immunoreactivity of CD9 suggests that CD9 still accumulates more abundantly around the disrupted paranodal region than in other parts of myelin, although the characteristic clusters are no longer present (Fig. $2 A, C$, arrows). The clustering of CD9 was occasionally observed at the paranode in the mutants (Fig. 2D,F, arrows) and colocalized with Caspr
(Fig. 2E,F, arrow). These results suggest that CD9 is one of the myelin components involved in paranodal junction formation.

\section{Lack of CD9 causes disruption of the paranodal junction in the CNS}

The localization of CD9 at the paranodal region raised the question of whether the lack of CD9 may affect the axoglial paranodal junction. CD9-deficient mice were created by several groups (Kaji et al., 2000; Le Naour et al., 2000; Miyado et al., 2000). These mice are healthy except for sperm-egg fusion. The life span of these mutants is normal, and for the nervous system, Terada et al. (2002) reported that oligodendrocyte production and myelination appear normal in this mutant. To examine the fine structure of the myelin membrane, we performed ultrastructural analyses on optic nerves from wild-type and CD9-deficient mice (Miyado et al., 2000) at 8 weeks of age. As shown in Figure $3 A$, the wildtype optic nerve showed that paranodal loops were arrayed sequentially and in close apposition to the axonal membrane. Periodic densities corresponding to the transverse bands were apparent between the loops and axolemma (Fig. 3C, arrows). In contrast, in the optic nerve of mice lacking CD9, the paranodal morphology was frequently perturbed, with most paranodal loops facing away from the axonal membrane (Fig. $3 B, D$ ) and transverse bands missing entirely (Fig. $3 D$ ), indicating that the axoglial junction was completely disrupted. Some loops occasionally retained a close association with the axon; however, even in these regions, transverse bands disappeared completely (Fig. $3 D$, arrows). Because CD9 is also located in the outermost surface of compact myelin (Nakamura et al., 1996), we examined semithin transverse sections of optic nerves from wild-type and CD9deficient mice with toluidine blue staining. No apparent abnormalities were observed in compact myelin in the mutant CNS (data not shown). These results demonstrate that CD9 plays a critical role in the apposition of paranodal loops to the axolemma and formation of the junction in CNS axons but not in the formation of compact myelin.

\section{Paranodal proteins were mislocalized in CD9-deficient mice}

Thus, our immunohistological and ultrastructural studies showed that CD9 was accumulated in the paranodal regions, and that the paranodal junctions were disrupted by the lack of CD9. To explore whether molecular rearrangements occur at the paranode, as shown in other paranodal mutants, we examined the distribution of the paranodal proteins Caspr and NF155 in CD9deficient mice. NF155 (green) and Caspr (red) were colocalized at the paranodal regions in optic nerves of wild-type mice (Fig. $4 A$ ). In contrast, in CD9-deficient mice, the distribution of NF155 and Caspr became diffuse in some axons, and the edges of positive staining were barely detectable in both the optic nerve (Fig. $4 B$ ) and the sciatic nerve (Fig. 4C). However, the frequency of such abnormalities was different among individuals and among axons even in the same mouse. To quantify Caspr clusters at the paranodal junction, we counted the number of Caspr clusters in the optic nerve and the sciatic nerve using $\mathrm{F}_{2}$ mice. The numbers were represented per field of view (FOV) (1 FOV, $48.7 \times 48.7 \mu \mathrm{m}^{2}$ ), and an average of the numbers in 10 FOVs was calculated from wild-type and CD9-deficient mice at 8 weeks of age. As shown in Table 1, the number of Caspr clusters in CD9deficient mice was reduced significantly to 75 and $47 \%$ compared with those of the wild type in the optic nerve and the sciatic nerve, respectively. The localization of voltage-gated ion channels was also examined. In the optic nerve (Fig. $4 D$ ) and the spinal root (Fig. $4 E$ ) from wild-type mice, $\mathrm{Na}^{+}$channels were concentrated 


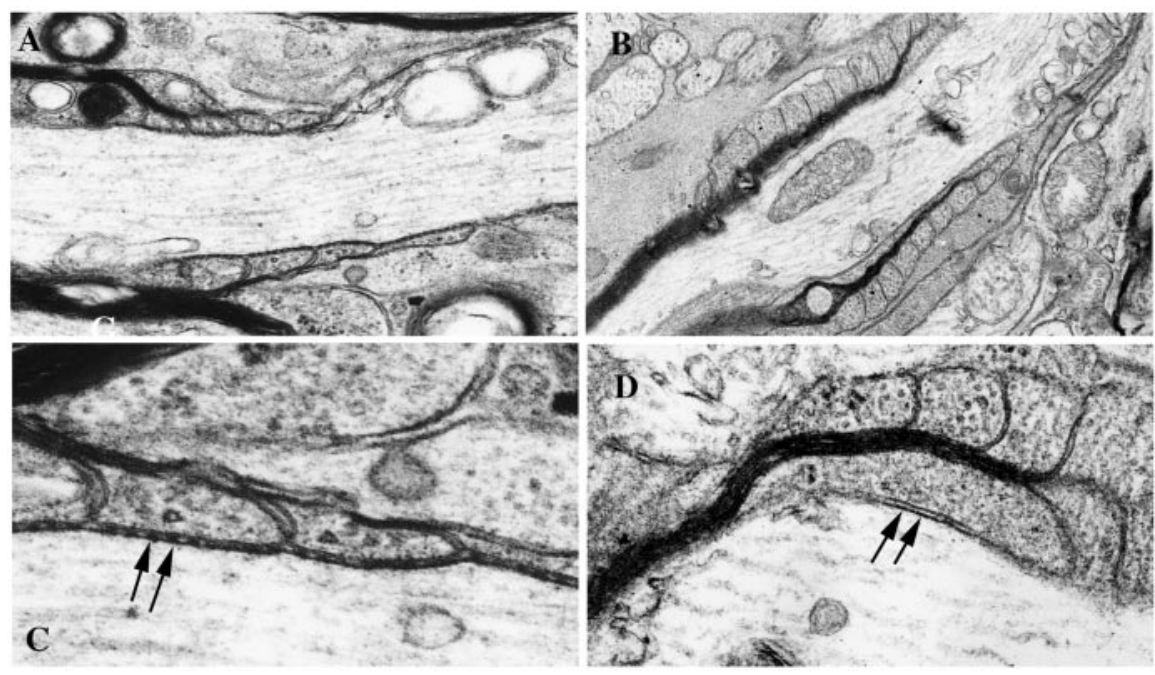

Figure 3. Ultrastructural analysis of the CNS nodal region. Shown are optic nerve longitudinal sections from 8-week-old wild-type ( $A$, $C$ and $C D$-deficient $(B, D)$ mice. In wild-type mice, the paranodal myelin loops are arrayed sequentially $(A)$, and the transverse bands between the loops and axolemma are clearly seen at high magnification ( $C$, arrows). In contrast, in CD9 mutant mice, the paranodal morphology is frequently perturbed. The paranodal loops face away from the axonal membrane $(B)$. The transverse bands are absent in such a paranode at high magnification ( $D$, arrows). $A, B, 12,000 \times$ magnification; $C, D, 30,000 \times$ magnification.
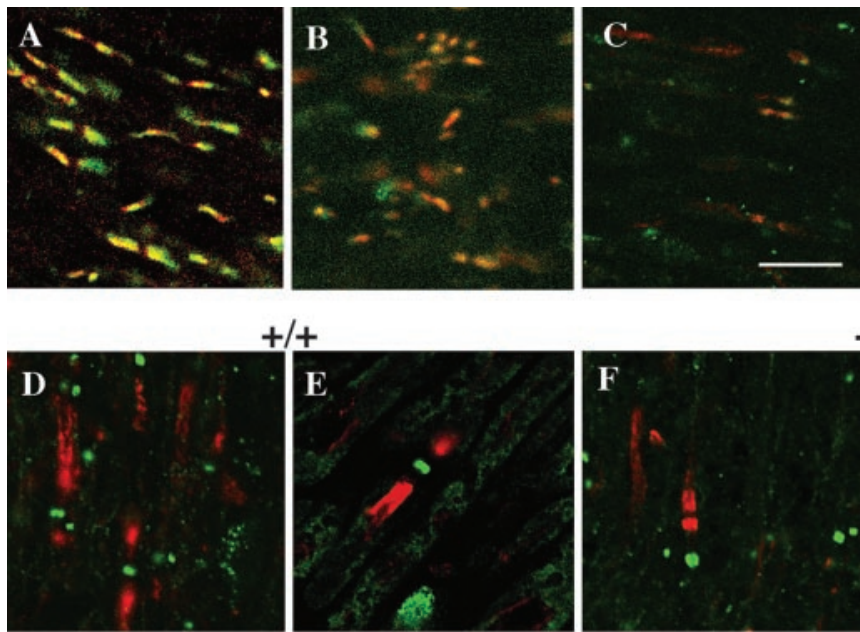

$++$
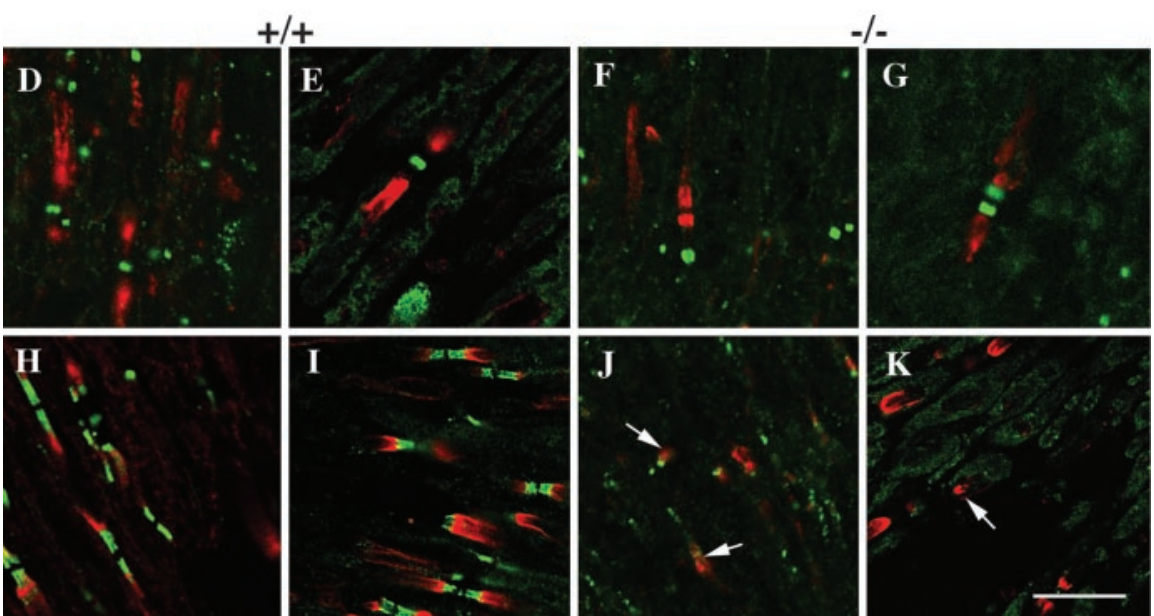

Figure 4. Distribution of nodal, paranodal, and juxtaparanodal components in 8-week-old mice. NF155 (green) and Caspr (red) are colocalized to form clusters at the paranode in the wild-type optic nerve (A). In contrast, in CD9 mutant mice, NF155 and Caspr become more diffusely distributed in some axons both in the optic nerve $(B)$ and in the sciatic nerve $(C)$. In wild-type mice $(D, E, H, I), \mathrm{Na}^{+}$channels $\left(D, E\right.$, green) cluster at the node, whereas $\mathrm{K}^{+}$channels $(D-I$, red) cluster at the juxtaparanode adjacent to the Caspr clusters $\left(H, I\right.$, green) both in the optic nerve $(D, H)$ and in the spinal root $(E, I)$. Compared with wild-type mice, $\mathrm{K}^{+}$ channels are mislocalized to the paranode $\left(G, J, K\right.$, arrows, red), and the shapes of $\mathrm{Na}^{+}$channel clusters are altered $(G$, green) and $(a s p r$ clusters have disappeared $(J, K$, green) in the optic nerve $(F, J)$ and the spinal root $(G, K)$ of CD9 mutant mice. Scale bars, $10 \mu \mathrm{m}$.

in the node of Ranvier, and the Kv1.1 potassium channel $\alpha$-subunit was localized at the juxtaparanodal region. In contrast, in CD9-deficient mice, irregular forms of $\mathrm{Na}^{+}$channel clusters, such as binary shape (Fig. 4G), were occasionally observed, and $\mathrm{K}^{+}$channel clusters frequently accumulated in the paranodal region adjacent to the $\mathrm{Na}^{+}$channel clusters (Fig. 4G). Further- more, in some CD9-deficient mice, $\mathrm{K}^{+}$ channel clusters were significantly decreased and hardly detected at the juxtaparanode both in the optic nerve (Fig. $4 J)$ and in the spinal root (Fig. $4 K$ ) compared with wild-type mice (Fig. $4 H, I$ ).

\section{CD9-deficient mice display}

hypermyelination in restricted fibers in the PNS

In contrast to the CNS axons, semithin transverse sections of sciatic nerves from CD9-deficient mice revealed axons with abnormally thick myelin (Fig. 5B). Interestingly, these affected axons were observed as a group that was surrounded by normal-appearing axons in the same sciatic nerve sections (Fig. 5B). Such thick myelin was never observed in wild-type mice (Fig. 5A). We calculated the ratio of axonal diameter to total fiber diameter ( $g$ values) of each axon in the randomly selected areas of these wild-type and CD9deficient sciatic nerves (Fig. 5C,D). As shown in Figure $5 C$, in wild-type nerves, the range of $g$ values settled from 0.55 to 0.83 , and the peak value was $0.69(n=$ $105)$. In contrast, in the mutant nerves (Fig. $5 D$ ), the $g$ ratio of each fiber varied, with a wide range from 0.08 to $0.79(n=$ 107 ), and the peak of the histogram was smaller (0.48) than that of wild-type nerves (0.69). This result indicated that the myelin thickness of the mutant nerves was thicker compared with wild-type nerves. Although transverse bands were found in most of the paranodes examined by electron microscopy (data not shown), the numbers of paranodes we observed were too limited to conclude whether the paranodal junction was preserved. Mislocalization of $\mathrm{K}^{+}$channels to the paranode in the PNS axons (Fig. 4) suggested disruption of the paranodal junction in some of these axons.

\section{CD9 is enriched in the PNS}

Thus, lack of CD9 influences myelin differently in the CNS and the PNS. Therefore we examined the content of CD9 protein in CNS and PNS myelin. From immunostaining patterns in longitudinal sections of spinal cords of wild-type mice, CD9 immunoreactivity in spinal roots (Fig. 6A,r) was much stronger than that in the spinal cord. Furthermore, in cross sections of spinal cords, paranodal regions of the CNS-PNS transitional zone (Fig. 6B, boxed area) were identified unmistakably by CD9, which was mainly detected in the peripheral side of dorsal roots (Fig. 6B, p). This transitional zone was determined by the presence of either myelin-oligodendrocyte glycoprotein (MOG) (CNS) or laminin (PNS) (data not shown). Figure 6C-E shows the higher magnifi- 
Table 1. Quantification of Caspr clusters in wild-type and CD9 mutant mice

\begin{tabular}{llc}
\hline & Wild-type mice & CD9 mutant mice \\
\hline CNS (optic nerves) & $52 \pm 7$ & $39 \pm 11^{*}$ \\
PNS (sciatic nerves) & $15 \pm 5$ & $7 \pm 6^{* *}$ \\
\hline
\end{tabular}

The normal-appearing Caspr clusters represented a pair of clusters (as indicated in Fig. 1B,E). The fluorescent intensity of these clusters was always $\geq 150$ using LSM5 Pascal software. The number of cluster pairs in 1 FOV $\left(48.7 \times 48.7 \mathrm{~mm}^{2}\right)$ was counted in the optic nerve and sciatic nerve sections in each mouse. At least three mice of each genotype were examined, and the mean \pm SEM number of normal Caspr cluster pairs is shown. ${ }^{*} p<0.05$ and ${ }^{* *} p<0.01$ (t test) compared with those in the wild-type mice.
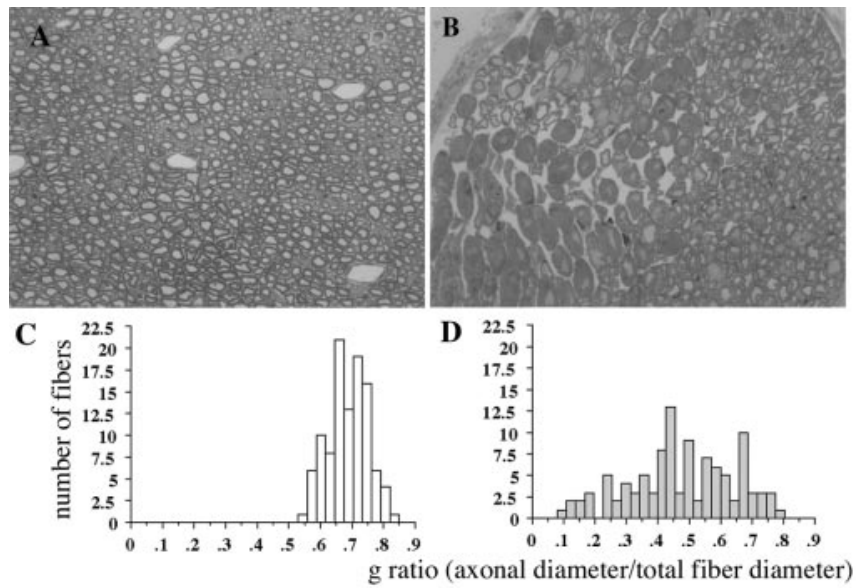

Figure 5. Analysis of PNS compact myelin. Compact myelin is severely affected in the PNS of the CD9 knock-out mouse. The transverse sections of sciatic nerves from wild-type $(A)$ and $C D 9$ mutant $(B)$ mice were stained with toluidine blue. Compared with wild type $(A)$, the group of axons with abnormally thick myelin are observed in the mutant $(B)$. These axons are gathered as a group and are surrounded by normal-appearing fibers ( $B$, right). $A, B, 40 \times$ magnification. $C, D$, Distribution of $g$ ratio (axonal diameter/total nerve diameter) in sciatic nerve fibers of wild-type $(n=105)$ and CD9 mutant $(n=107)$ mice.

cation of the boxed area in Figure $6 B$, with double staining of Caspr (red) and CD9 (green). As shown in Figure 6, $C$ and $E$, Caspr was clustered at the paranode both in the CNS (arrowhead) and in the PNS (arrow). The asterisk indicates the node of Ranvier in the CNS-PNS transition. In contrast, CD9 clusters were easily detected in the PNS paranode (Fig. $6 D$, arrow) but not in the CNS (Fig. 6D, arrowhead). This result suggested that although CD9 was localized both in the PNS and in the CNS paranode, the amount of this protein was significantly different. Such differences were also observed at the CNS-PNS transitional zone in the ventral roots (data not shown). Western blot analysis of brain myelin fraction and sciatic nerve homogenate confirmed that CD9 was more highly enriched in the PNS (Fig. 6F).

\section{Discussion}

To learn more about how myelin-forming glia affect the morphology of the paranode, we focused on the CD9 glial molecule. In this report, we demonstrate that a glial protein other than adhesion molecules contributes to paranode formation.

\section{Possible roles of CD9 at the paranode}

In this study, we showed that CD9 is a paranodal protein in glia. From recent studies using CST-deficient mice, it is certain that sulfatide is an essential component for the paranodal junction (Honke et al., 2002; Ishibashi et al., 2002). Because CD9 in this mutant was no longer concentrated at the paranode (Fig. $2 A-F$ ), sulfatide may contribute to CD9 accumulation at the paranode. Yashiro-Ohtani et al. (2000) suggested that CD9 is localized in lipid raft domains on the plasma membrane. Although it is still possible that the disruption of the paranodal junction itself in CST-deficient mice causes the mislocalization of CD9, our observations suggest that CD9 is incorporated into the lipid rafts in the presence of sulfatide and is transported to the paranodal loops.

From immunohistochemical studies using CD9-deficient mice, we clearly demonstrated that CD9 is important for the formation of paranodal junctions both in the CNS and in the PNS. First, the paranodal junctions were disrupted, as shown by the disappearance of transverse bands in the CNS (Fig. 3B,D). Second, Caspr clusters were distributed diffusely on the axon both in the CNS and in the PNS (Fig. $4 B, C, J, K$ ). Third, $\mathrm{K}^{+}$channels clustered at the region adjacent to $\mathrm{Na}^{+}$channel clusters (Fig. $4 G$ ), as shown in other paranodal mutants (Dupree et al., 1999; Bhat et al., 2001; Boyle et al., 2001; Ishibashi et al., 2002).

What is the functional role of CD9 at the paranode? No data suggest that CD9 itself is an adhesion molecule. A characteristic feature of tetraspanins, including CD9, is to form complexes with a variety of membrane proteins at the plasma membrane. They are involved in numerous cellular processes, including cellular activation, motility, metastasis, and tumor-suppression activities (for review, see Berditchevski, 2001; Hemler, 2001). Their apparent diversity of functions, the lack of strong phenotypes associated with the elimination of single tetraspan genes, and the data suggesting that tetraspanins can act as adjunct but nonessential components of signaling complexes led to their designation as molecular facilitators (Maecker et al., 1997). Therefore, it is difficult to conclude that CD9 at the paranode works as a direct ligand of the axonal molecules. It is more likely that CD9 may have some role in modulating other paranodal molecules by binding to form membrane protein complexes. Interestingly, CD9 is known to bind with the membrane-anchored form of heparin-binding EGF-like growth factor and upregulates its binding activity to the ligand (Iwamoto et al., 1994; Higashiyama et al., 1995; Nakamura et al., 2000). A glial adhesion molecule NF155 is present at the paranode (Tait et al., 2000). Such localization of NF155 occurs after Caspr clustering (Marcus et al., 2002), and this protein is now known to be a glial receptor for Caspr-contactin complex at the paranodal axolemma (Charles et al., 2002). However, both in Caspr-deficient mice and in contactin-deficient mice, some NF155 is still detected at the paranode (Bhat et al., 2001; Boyle et al., 2001); therefore, the presence of these axonal ligands is not essential for the targeting of NF155 to the glial paranodal loops. It is likely that other glial molecules such as CD9 are involved in this process. As described above, CD9 complex may be incorporated into microdomains where specific lipids like sulfatide are present. Thus, it is possible that CD9 contributes to paranodal junctional formation by regulating binding activity of glial molecules such as NF155 to axonal ligands, by transporting these glial molecules to accumulate in the paranode, or both.

\section{Differences found in CD9 mutant mice compared with other paranodal mutants}

As described previously, CD9-deficient mice showed alterations of Caspr and $\mathrm{K}^{+}$channel localization similar to those observed in other paranodal mutants. However, there were some differences found in the CD9 mutants. Although all of the paranodal mutants displayed severe neurological deficits, CD9-deficient mice displayed no apparent neurological deficit, and their life span was normal. The difference in the phenotype may be attributable to the severity of affected nerves in CD9-deficient mice. The frequency of the abnormalities of axonal protein localizations was less in CD9-deficient mice compared with other paranodal mutants. In peripheral compact myelin of CD9-deficient mice, affected axons were observed as a group surrounded by normal- 


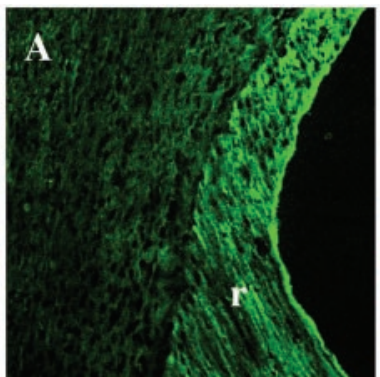

B
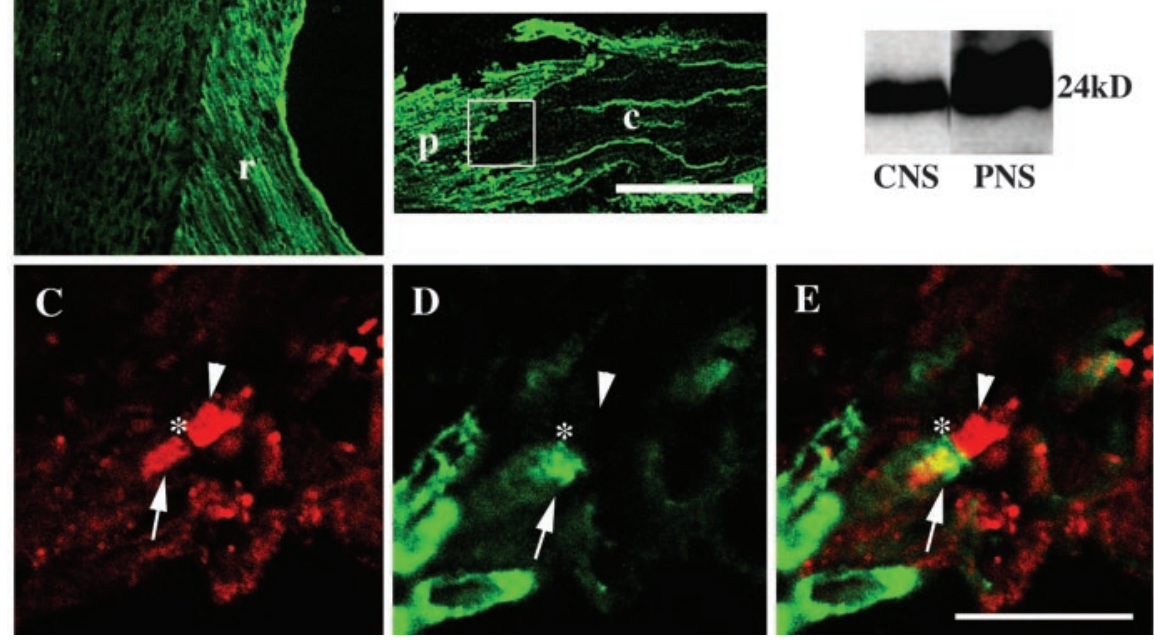

Figure 6. CD9 is more highly enriched in the PNS than in the CNS. Longitudinal sections of the spinal cord with the root $(A)$ and $\operatorname{dorsal} \operatorname{root}(B)$ from 8-week-old mice were stained with anti-CD9 antibody. $A$, The intensity of $C D 9$ staining in the spinal root (r) is stronger than that in the spinal cord. $B$, The transitional zone between the CNS (c) and the PNS ( $\mathrm{p}$ ) in the dorsal root indicated by the boxed area is clearly demarcated by CD9 immunostaining. This zone was determined by the presence of myelin- oligodendrocyte glycoprotein (CNS) or laminin (PNS) (data not shown). $(-E$, Higher magnification of the area indicated by the box in B. Using a cenvical spinal cord cross section, double immunostaining of Caspr (red) and CD9 (green) was performed. The transitional zone is shown at higher magnification, and the asterisks indicate the node of Ranvier. Caspr forms clusters atboth paranodes $(C, E)$, whereas $C D 9$ isidentified only in thePNS paranode $(D, E$, arrow) butnot in the CNS paranode (arrowhead). From the Westem blot analysis, the amount of CD9 is significantly higher in the PNS homogenate ( $F$. Scale bars: $A, B, 100 \mu \mathrm{m} ; C-E, 10 \mu \mathrm{m}$.

reports about the function of CD9 in Schwann cell proliferation (Hadjiargyrou and Patterson, 1995) and migration (Anton et al., 1995), no evidence has been found previously that CD9 can regulate myelination. Therefore, this study is the first report to show the functional role of CD9 in myelination in vivo. It is possible that myelination is terminated by CD9 together with some other molecules, including integrins and extracellular matrix proteins. Because CD9 interacts with several molecules, including the $\beta_{1}$ integrin subfamily (Nakamura et al., 1995; Berditchevski et al., 1996; Schmidt et al., 1996; Terada et al., 2002), and because Schwann cells express $\beta_{1}$ integrin (Salzer, 1995), $\beta_{1}$ integrin is one of the candidates for this function. The $\beta_{1}$ integrin subfamily comprises heterodimeric transmembrane proteins that mediate cell adhesion to extracellular matrix proteins, including laminin $\left(\alpha_{3} \beta_{1}\right.$, $\left.\alpha_{6} \beta_{1}\right)$, collagen $\left(\alpha_{1} \beta_{1}, \alpha_{2} \beta_{1}, \alpha_{3} \beta_{1}\right)$, and fibronectin $\left(\alpha_{4} \beta_{1}, \alpha_{5} \beta_{1}\right)$ (Hynes, 1992). In the PNS, a basal lamina surrounding Schwann cells contains laminin; therefore, the appearance of CD9 together with $\beta_{1}$ integrin on the myelin surface may mediate a termination of myelination signal by binding to laminin.

appearing axons in the same section (Fig. 5B). Thus, these intact fibers may be enough to prevent neurological symptoms.

So why are only limited numbers of axons affected in CD9deficient mice? As described above, tetraspanins interact with each other. Latebloomer, the first identified Drosophila tetraspanin gene (Kopczynski et al., 1996), is expressed on motoneurons and contributes to the establishment of synaptic contacts at the neuromuscular junction in company with at least two other tetraspanins (Fradkin et al., 2002). Therefore, at the axoglial junction in mammals, several tetraspanins, including CD9, may be involved and may compensate for the loss of CD9 in the mutant. This may explain the varieties of phenotypes among individuals as well as axons in the same mouse. The influence of genetic background may also explain variations among individuals, because the findings became less prominent in the $\mathrm{F}_{3}$ and $\mathrm{F}_{4}$ generations of $\mathrm{C} 57 \mathrm{BL} / 6 \mathrm{~J}$ mice compared with those of the $\mathrm{F}_{2}$ generation.

CD9 may act as the terminal signal of myelination in the PNS The thickness of the myelin sheath around an axon is important in determining conduction velocity. Theoretically, the maximal value of $\lambda$, the space constant, is achieved when the ratio $g$ (i.e., axonal diameter divided by the total diameter) is $\sim 0.6$. Actually, most myelinated fibers in the PNS have $g$ values between 0.47 and 0.74 (Ritchie, 1995), suggesting that there should be signals for terminating myelination. Because CD9 is distributed along the outermost membrane of compact myelin (Nakamura et al., 1996) and appears at the end of the myelination process (Kagawa et al., 1997), it is possible that CD9 may be such a signal. Severe hypermyelination was observed in the mutant sciatic nerves. Interestingly, hypermyelinating axons were observed as a group that was surrounded by normal-appearing myelinated axons in the same sciatic nerve sections. Nevertheless, although there are several
Although CD9 is present at the outer surface of myelin both in the CNS and in the PNS, the thickness of compact myelin in the CNS was not affected in CD9 mutants. This may be attributable to a difference in myelin-forming cells between the CNS and the PNS. In the CNS, processes from a single oligodendrocyte wrap layers around several axons, whereas each Schwann cell forms myelin around only a single axon in the PNS. Furthermore, because the amount of CD9 in the CNS is much less than that in the PNS, other molecules may be involved. The fact that MOG is also located at the external surfaces of myelin sheaths in the CNS and appears at a later stage of myelination suggests that this protein may function as a termination signal for CNS myelin (Brunner et al., 1989; Dyer and Matthieu, 1994). We also found that CD81, another member of the tetraspanins, was enriched in CNS myelin; therefore, different tetraspanins may work individually in the CNS and the PNS (our unpublished data). On the basis of our present experiments, additional studies of the molecular basis of the mechanisms of CD9 intracellular and extracellular signals will provide important clues about the molecular basis underlying the control of myelination in the PNS.

\section{References}

Anton ES, Hadjiargyrou M, Patterson PH, Matthew WD (1995) CD9 plays role in Schwann cell migration in vitro. J Neurosci 15:584-595.

Baumgartner S, Littleton JT, Broadie K, Bhat MA, Harbecke R, Lengyel JA, Chiquet-Ehrismann R, Prokop A, Bellen HJ (1996) A Drosophila neurexin is required for septate junction and blood-nerve barrier formation and function. Cell 87:1059-1068.

Berditchevski F (2001) Complexes of tetraspanins with integrins: more than meets the eye. J Cell Sci 114:4143-4151.

Berditchevski F, Zutter MM, Hemler ME (1996) Characterization of novel complexes on the cell surface between integrins and proteins with 4 transmembrane domains (TM4 proteins). Mol Biol Cell 7:193-207.

Bhat MA, Rios JC, Lu Y, Garcia-Fresco GP, Ching W, St Martin M, Li J, Einheber S, Chesler M, Rosenbluth J, Salzer JL, Bellen HJ (2001) Axon- 
glia interactions and the domain organization of myelinated axons requires neurexin IV/Caspr/Paranodin. Neuron 30:369-383.

Boucheix C, Benoit P (1988) CD9 antigen: will platelet physiology help to explain the function of a surface molecule during hemopoietic differentiation? Nouv Rev Fr Hematol 30:201-202.

Boyle MET, Berglund EO, Murai KK, Weber L, Peles E, Ranscht B (2001) Contactin orchestrates assembly of septate-like junctions at the paranode in myelinated peripheral nerve. Neuron 30:385-397.

Brunner C, Lassmann H, Waehneldt TV, Matthieu JM, Linington C (1989) Differential ultrastructural localization of myelin basic protein, myelin/ oligodendroglial glycoprotein, and $2^{\prime}, 3^{\prime}$-cyclic nucleotide $3^{\prime}$ phosphodiesterase in the CNS of adult rats. J Neurochem 52:296-304.

Charles P, Tait S, Faivre-Sarrailh C, Barbin G, Gunn-Moore F, DenisenkoNehrbass N, Guennoc AM, Girault JA, Brophy PJ, Lubetzki C (2002) Neurofascin is a glial receptor for the paranodin/Caspr-contactin axonal complex at the axoglial junction. Curr Biol 12:217-220.

Dupree JL, Girault J-A, Popko B (1999) Axo-glial interactions regulate the localization of axonal paranodal proteins. J Cell Biol 147:1145-1151.

Dyer CA, Matthieu JM (1994) Antibodies to myelin/oligodendrocytespecific protein and myelin/oligodendrocyte glycoprotein signal distinct changes in the organization of cultured oligodendroglial membrane sheets. J Neurochem 62:777-787.

Einheber S, Zanazzi G, Ching W, Scherer S, Milner TA, Peles E, Salzer JL $(1997)$ The axonal membrane protein Caspr, a homologue of neurexin IV, is a component of the septate-like paranodal junctions that assemble during myelination. J Cell Biol 139:1495-1506.

Faivre-Sarrailh C, Gauthier F, Denisenko-Nehrbass N, Bivic A, Rougon G, Girault JA (2000) The glycosylphosphatidyl inositol-anchored adhesion molecule F3/contactin is required for surface transport of paranodin/ contactin-associated protein (caspr). J Cell Biol 149:491-501.

Fradkin LG, Kamphorst JT, DiAntonio A, Goodman CS, Noordermeer JN (2002) Genomewide analysis of the Drosophila tetraspanins reveals a subset with similar function in the formation of the embryonic synapse. Proc Natl Acad Sci USA 99:13663-13668.

Hadjiargyrou M, Patterson PH (1995) An anti-CD9 monoclonal antibody promotes adhesion and induces proliferation of Schwann cells in vitro. J Neurosci 15:574-583.

Hemler ME (2001) Specific tetraspanin functions. J Cell Biol 155:1103-1107.

Higashiyama S, Iwamoto R, Goishi K, Raab G, Taniguchi N, Klagsbrun M, Mekada E (1995) The membrane protein CD9/DRAP27 potentiates the juxtacrine growth factor activity of the membrane-anchored heparinbinding EGF-like growth factor. J Cell Biol 128:929-938.

Honke K, Hirahara Y, Dupree J, Suzuki K, Popko B, Fukushima J, Nagasawa T, Yoshida N, Wada Y, Taniguchi N (2002) Paranodal junction formation and spermatogenesis require sulfoglycolipids. Proc Natl Acad Sci USA 99:4227-4232.

Huber LA, Madison DL, Simons K, Pfeiffer SE (1994) Myelin membrane biogenesis by oligodendrocytes: developmental regulation of lowmolecular weight GTP-binding proteins. FEBS Lett 347:273-278.

Hynes RO (1992) Integrins: versatility, modulation, and signaling in cell adhesion. Cell 69:11-25.

Ishibashi T, Dupree JL, Ikenaka K, Hirahara Y, Honke K, Peles E, Popko B, Suzuki K, Nishino N, Baba H (2002) A myelin galactolipid, sulfatide, is essential for maintenance of ion channels on myelinated axon but not essential for initial cluster formation. J Neurosci 22:6507-6514.

Iwamoto R, Senoh H, Okada Y, Uchida T, Mekada E (1991) An antibody that inhibits the binding of diphtheria toxin to cells revealed the association of a $27-\mathrm{kDa}$ membrane protein with the diphtheria toxin receptor. J Biol Chem 266:20463-20469.

Iwamoto R, Higashiyama S, Mitamura T, Taniguchi N, Klagsbrun M, Mekada E (1994) Heparin-binding EGF-like growth factor, which acts as the diphtheria toxin receptor, forms a complex with membrane protein DRAP27/CD9, which up-regulates functional receptors and diphtheria toxin sensitivity. EMBO J 13:2322-2330.

Kagawa T, Mekada E, Shishido Y, Ikenaka K (1997) Immune system-related CD9 is expressed in mouse central nervous system myelin at a very late stage of myelination. J Neurosci Res 50:312-320.

Kaji K, Oda S, Shikano T, Ohnuki T, Uematsu Y, Sakagami J, Tada N, Miyazaki S, Kudo A (2000) The gamete fusion process is defective in eggs of Cd9-deficient mice. Nat Genet 24:279-282.

Kim T, Fiedler K, Madison DL, Krueger WH, Pfeiffer SE (1995) Cloning and characterization of MVP17: a developmentally regulated myelin protein in oligodendrocytes. J Neurosci Res 42:413-422.

KopczynskiCC, Davis GW, Goodman CS (1996) A neural tetraspanin, encoded by late bloomer, that facilitates synapse formation. Science 271:1867-1870.

Le Naour F, Rubinstein E, Jasmin C, Prenant M, Boucheix C (2000) Severely reduced female fertility in CD9-deficient mice. Science 287:319-321.

Maecker HT, Todd SC, Levy S (1997) The tetraspanin superfamily: molecular facilitator. FASEB J 11:428-442.

Marcus J, Dupree JL, Popko B (2002) Myelin-associated glycoprotein and myelin galactolipids stabilize developing axo-glial interactions. J Cell Biol 156:567-577.

Menegoz M, Gaspar P, Le Bert M, Galvez T, Burgaya F, Palfrey C, Ezan P, Arnos F, Girault JA (1997) Paranodin, a glycoprotein of neuronal paranodal membranes. Neuron 19:319-331.

Miyado K, Yamada G, Yamada S, Hasuwa H, Nakamura Y, Ryu F, Suzuki K, Kosai K, Inoue K, Ogura A, Okaba M, Mekada E (2000) Requirement of CD9 on the egg plasma membrane for fertilization. Science 287:321-324.

Nakamura K, Mitamura T, Takahashi T, Kobayashi T, Mekada E (2000) Importance of the major extracellular domain of CD9 and the epidermal growth factor (EGF)-like domain of heparin-binding EGF-like growth factor for upregulation of binding and activity. J Biol Chem 275:18284-18290.

Nakamura Y, Iwamoto R, Mekada E (1995) Membrane-anchored heparinbinding EGF-like growth factor (HB-EGF) and diphtheria toxin receptorassociated protein (DRAP27)/CD9 form a complex with integrin alpha 3 beta 1 at cell-cell contact sites. J Cell Biol 129:1691-1705.

Nakamura Y, Iwamoto R, Mekada E (1996) Expression and distribution of CD9 in myelin of the central and peripheral nervous systems. Am J Pathol 149:575-583.

Norton WT, Poduslo SE (1973) Myelination in rat brain: method of myelin isolation. J Neurochem 21:749-757.

Pedraza L, Huang JK, Colman DR (2001) Organizing principles of the axoglial apparatus. Neuron 30:335-344.

Peles E, Nativ M, Lustig M, Grumet M, Schilling J, Martinez R, Plowman GD, Schlessinger J (1997) Identification of a novel contactin-associated transmembrane receptor with multiple domains implicated in proteinprotein interactions. EMBO J 16:978-988.

Rasband MN, Trimmer JS, Schwarz TL, Levinson SR, Ellisman MH, Schachner M, Shrager P (1998) Potassium channel distribution, clustering, and function in remyelinating rat axons. J Neurosci 18:36-47.

Rios JC, Melendez-Vasquez CV, Einheber S, Lustig M, Grumet M, Hemperly J, Peles E, Salzer JL (2000) Contactin-associated protein (Caspr) and contactin from a complex that is targeted to the paranodal junctions during myelination. J Neurosci 20:8354-8364.

Ritchie JM (1995) Physiology of axons. In: The axon (Waxman SG, Kocsis JD, Stys PK, eds), pp 68-93. New York: Oxford UP.

Rosenbluth J (1976) Intramembranous particle distribution at the node of Ranvier and adjacent axolemma in myelinated axons of the frog brain. J Neurocytol 5:731-745.

Salzer JL (1995) Mechanisms of adhesion between axons and glial cells. In: The axon (Waxman SG, Kocsis JD, Stys PK, eds), pp 164-184. New York: Oxford UP.

Sato T (1968) A modified method for lead staining of thin sections. J Electron Microsc 17:158-159.

Schmidt C, Kunemund V, Wintergerst ES, Schmitz B, Schachner M (1996) CD9 of mouse brain is implicated in neurite outgrowth and cell migration in vitro and is associated with the $\alpha 6 / \beta 1$ integrin and the neural adhesion molecule L1. J Neurosci Res 43:12-31.

Tait S, Gunn-Moore F, Collinson JM, Huang J, Lubetzki C, Pedraza L, Sherman DL, Colman DR, Brophy PJ (2000) An oligodendrocyte cell adhesion molecule at the site of assembly of the paranodal axo-glial junction. J Cell Biol 150:657-666.

Terada N, Baracskay K, Kinter M, Melrose S, Brophy PJ, Boucheix C, Bjartmar C, Kidd G, Trapp BD (2002) The tetraspanin protein, CD9, is expressed by progenitor cells committed to oligodendrogenesis and is linked to betal integrin, CD81, and Tspan-2. Glia 40:350-359.

Trimmer JS (1991) Immunological identification and characterization of a delayed rectifier $\mathrm{K}^{+}$channels polypeptide in rat brain. Proc Natl Acad Sci USA 88:10764-10768.

Wright MD, Tomlinson MG (1994) The ins and outs of the transmembrane 4 superfamily. Immunol Today 15:588-594.

Yashiro-Ohtani Y, Zhou X, Toyo-oka K, Tai X, Park C, Hamaoka T, Abe R, Miyake K, Fujiwara F (2000) Non-CD28 costimulatory molecules present in $\mathrm{T}$ cell rafts induce $\mathrm{T}$ cell costimulation by enhancing the association of TCR with rafts. J Immunol 164:1251-1259. 